\title{
Efficient Interactive Pre-integrated Volume Rendering
}

\author{
Heewon Kye ${ }^{1,3}$, Helen Hong ${ }^{2}$, and Yeong Gil Shin ${ }^{1,3}$ \\ ${ }^{1}$ School of Computer Science and Engineering, Seoul National University \\ \{kuei, yshin\}@cglab.snu.ac.kr \\ ${ }^{2}$ School of Computer Science and Engineering, BK21: Information Technology, Seoul \\ National University, San 56-1 Shinlim-dong Kwanak-gu, Seoul 151-742, Korea \\ hlhong@cse.snu.ac.kr \\ ${ }^{3}$ INFINITT Co., Ltd., Taesuk Bld., 275-5 Yangjae-dong Seocho-gu, Seoul 137-934, Korea
}

\begin{abstract}
Pre-integrated volume rendering has become one of the most efficient and important techniques in three dimensional medical visualization. It can produce high-quality images with less sampling. However, two important issues have received little attention throughout the ongoing discussion of preintegration: Skipping over empty-space and the size of lookup table for a transfer function. In this paper, we present a novel approach for empty-space skipping using the overlapped-min-max block. Additionally, we propose a new approximation technique to reduce the dependent texture size so that it decreases the size of texture memory and the update time. We demonstrate performance gain and decreasing memory consumption for typical renditions of volumetric data sets.
\end{abstract}

\section{Introduction}

Pre-integrated volume rendering is a technique for reconstructing the continuous volume rendering integral. Utilizing a pre-processed look-up table (called preintegration table), this method not only eliminates a lot of artifacts but also reduces the sampling rate for rendering. However, since this method uses two consecutive sample values as an index for the pre-integration table which is constructed before rendering for a given classified function, conventional acceleration techniques such as empty space skipping or interactive classification methods are not applied as it is.

Skipping empty space has been extensively exploited to accelerate volume rendering. However, pre-integrated volume rendering samples two consecutive points as a line segment, previous empty-space skipping methods could not be directly applied. The pre-integration table is indexed by three integration parameters: two consecutive sample values and the distance between those samples. To accelerate the pre-integration step, Engel et al. reduced the dimensionality of the table from three to two by summing a constant sampling distance [1]. Even though they used a twodimensional pre-integration table, it is still bulky when rendering high-precision data such as 12 bits-per-voxel data which is common in medical applications. A 12-bit image requires 256 times more memory and updating time than an 8-bit image.

In this paper, we present a novel data structure, called the overlapped-min-max block for applying empty-space scheme to the pre-integrated volume rendering, and a new approximation technique for reducing the dimensionality of the table from two to 
one. We implement them on recent consumer graphics hardware and on software-only shear-warp rendering [2] and ray-casting [3]. With our accelerations, the rendering and classification speed is much faster for medical datasets while maintaining the image quality.

\section{Overlapped Min-max Block for Empty-Space Skipping}

Traditional rendering methods sample a value at a point in three-dimensional space to get a color and opacity. If a block is entirely transparent, additional samplings in the block can be skipped. Pre-integrated volume rendering samples two points to get their color and opacity. Since two sampling points form a line segment, or a slab, all the blocks that intersect the line segment should be transparent for skipping the sampling process. As shown in Fig. 1, a line segment may intersect at most three blocks in twodimensional representation. Retrieving information three-times from the lookup table degrades the rendering performance. In addition, there is an overhead to determine which blocks are transparent (there are two cases such as Fig. 1a and Fig. 1b).

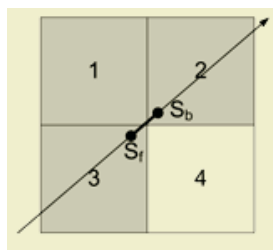

(a)

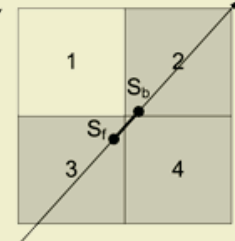

(b)

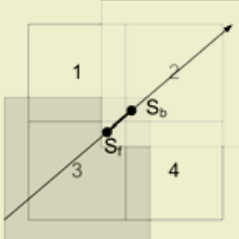

(c)

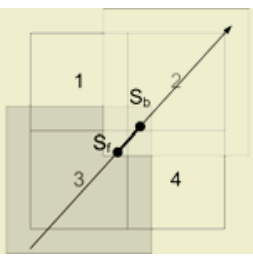

(d)

Fig. 1. The overlapped min-max table for pre-integrated volume rendering. There are two sampling point $S_{f}$ and $S_{b}$, and four blocks from block1 to block4. $\mathrm{S}_{\mathrm{f}}$ is in block3 and $S_{b}$ is in block2. To skip the line segment $S_{f} S_{b}$, (a) block1, block2, and block3 have to be transparent, (b) block2, block3, and block4 have to be transparent in the previous block structure. Moreover, there is overhead to determine the current situation is either (a) or (b). In our method, the overlapped-min-max block, each block holds some region jointly with its neighbors such as (c) and (d). To skip the line segment, we can test only one block, block2 (or block3)

Making each line segment belonging to only single block, we can efficiently decide whether we skip or not by testing one block. For this, we modify the region covered by each block. Each block covers some region of which thickness is at least the sampling distance as shown in Fig. 1c and Fig. 1d. By overlapping the region of each block, we can easily test whether the block of a line segment is transparent using only that block. This scheme is especially efficient on graphics hardware for its simplicity.

\section{Efficient Pre-integration Table}

In accelerated pre-integrated rendering, opacity of $i$-th sample $\left(\alpha_{i}\right)$ is written as:

$$
\begin{aligned}
& 1-\alpha_{i}=1-\alpha\left(s_{f}, s_{b}\right) \approx \exp \left(-\frac{1}{s_{b}-s_{f}}\left(T\left(s_{b}\right)-T\left(s_{f}\right)\right)\right) \\
& \text { where, } T(s):=\int_{0}^{s} \tau(s) d s .
\end{aligned}
$$


Because equation (1) comprehends the ray segment integral of a transfer function, we do not need to consider the maximum of the Nyquiest frequencies of the transfer functions $\tau(s)$ with the scalar field $s$. Therefore, it is sufficient to sample a volume with relatively low frequency. The 2D lookup table to obtain $\alpha_{i}$, requires a texture of which size is $N^{2}$, where $N$ is density range. In order to generate color images, each texture entry requires 4 bytes. When an image has 12-bit pixel depth, the required texture size becomes $4096^{2}$ and the required memory is $4096^{2} \times 4$ byte $=64 \mathrm{MB}$. The bigger lookup table brings the longer generation time and lower cache-efficiency. In this paper, we propose an 1D-texture lookup method that needs only 4096 entries. Because scalar values $s$ are usually quantized equation (1) can be rewritten as:

$$
\begin{aligned}
& \left.1-\alpha_{i} \approx s_{b}-s_{f}\right] \exp \left(-\sum_{s=s_{f}}^{s_{b}} \tau(s)\right) \\
& \text { where, } \quad S(x)=\sum_{s=0}^{x} \exp (-\tau(s)), S(-1)=0 .
\end{aligned}
$$

We modify a geometric average of transparency, into an arithmetic average as shown in equation (2). Therefore, only 1D-texture $S(x)$ needs to be stored to get the $\alpha_{i}$. The color can be formulized in a similar fashion.

\section{Experimental Results}

Experiments have been performed on a PC equipped with Pentirum $42.8 \mathrm{GHz}$ processor, 1GB main memory and an ATI 9800. Table 1 summarizes the performance using empty-space skipping (ESP). Obviously, skipping empty-space gains more performance. In hardware rendering, rendering time is reduced two times or more by using ESP. In software rendering, the gain of ESP is much bigger (10-30 times faster than w/o ESP). Since the angio dataset contains more empty space than the head dataset, the performance improvement factor of the angio dataset is bigger than that of the head dataset.

Table 1. A performance comparison in case of using empty-space skipping (msec)

\begin{tabular}{lcccccc}
\hline Dataset & \multicolumn{2}{c}{ 3D Hardware } & \multicolumn{2}{c}{ Ray-Casting } & \multicolumn{2}{c}{ Shear-Warp } \\
\hline & with ESP & w/o ESP & with ESP & w/o ESP & with ESP & w/o ESP \\
\hline BigHead & 83.8 & 302.1 & 897.7 & 12409 & 747.6 & 7904 \\
Angio & 95.1 & 392.1 & 737.6 & 16183 & 339.6 & 9984 \\
\hline
\end{tabular}

If a transfer function is fixed using 2D-texture (88.5ms) slightly faster than our 1Dtexture $(95.1 \mathrm{~ms})$ since $1 \mathrm{D}$-texture requires two texture loads for classification while 2D-texture requires one. However, when the transfer function is changed, using our 1D-texture $(101.2 \mathrm{~ms})$ is much faster than 2D-texture $(824.4 \mathrm{~ms})$ because of lookup table creation time. Fig. 2 show rendered images of BigHead and Angio volume data, respectively. There is no noticeable difference between using 1D-texture and 2Dtexture. 


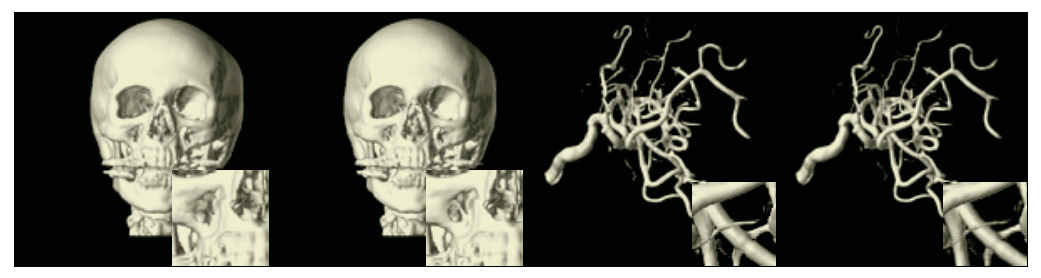

Fig. 2. The comparison of image quality for BigHead and Angio volume using (left) 1Dtexture, (right) and 2D-texture

\section{Conclusions}

In this paper, we have proposed a new method to accelerate traversal and classification of both hardware and software based pre-integrated volume rendering. Using the overlapped-min-max block, empty-space skipping can be accomplished more efficiently and can be easily implemented in a hardware-based method. To reduce the classification time and memory consumption, a new approximation method of a lookup table is also proposed. With regard to image quality, we have presented the minimum bound of error theoretically. Experimental results show that our method produces the same quality of rendered images as the previous classification method of pre-integration.

\section{References}

1. K. Engel, M. Kraus, and T. Ertl. High-Quality Pre-Integrated Volume Rendering Using Hardware-Accelerated Pixel Shading. Eurographics / SIGGRAPH Workshop on Graphics Hardware, Los Angeles, CA, USA, August 2001.

2. P. Lacrout and M. Levoy, Fast Volume Rendering Using a Shear-Warp Factorization of the Viewing Transformation. Proceedings of SIGGRAPH 94, 451-458, 1994.

3. M. Levoy, Display of Surfaces from Volume Data, IEEE Computer Graphics \& Application, 8: 29-37, 1988. 